\title{
PRECISION PLOT SEEDER FOR MEDICINAL AND AROMATIC PLANTS - CONCEPT, DEVELOPMENT AND OPTIMISATION
}

\author{
Author(s): \\ Z. Gobor ${ }^{1}-$ H. Heuberger ${ }^{2}-$ R. Rinder ${ }^{2}$
}

Affiliation:

'Institute for Agricultural Engineering and Animal Husbandry (ILT), Bavarian State Research Center for Agriculture (LfL), Vöttinger Str. 36, Freising, D-85354, Germany

${ }^{2}$ Institute for Crop Science and Plant Breeding (IPZ), Bavarian State Research Center for Agriculture (LfL),

Am Gereuth 2, Freising, D-85354, Germany

Email address:

zoltan.gobor@LfL.bayern.de, heidi.heuberger@|fl.bayern.de,rudolf.rinder@|fl.bayern.de

\begin{abstract}
A novel precision plot seeder for medicinal and aromatic plants was designed and built. Based on the system design-oriented development as well as the implemented state-of-the-art hardware and software solutions, it was possible to identify the problems in the early phase of the design process, to create multiple design variants, to overcome the lack of cross-disciplinary knowledge and consequently achieve savings in associated labour and material costs. The electrification should provide more accurate seed placement and continuous regulation of seed rates and row length for each row, as well as the option to generate detailed documentation about the sowing process.
\end{abstract}

\section{Keywords}

Precision plot seeder, experimental practice, digital prototyping, system based designs

\section{Introduction}

The Food and Agriculture Organisation of the United Nations (FAO) projected that if current patterns in food consumption will persist, 60 percent more food will need to be produced to meet the needs of the growing earth population that is expected to top the nine billion mark in 2050. Changes towards more sustainable production are required including investments in research, development and dissemination of the technologies and information in order to improve efficiency and reduce waste and pollution. The increase of the resource use efficiency, cutting the use of fossil fuels and reducing direct environmental degradation are some of the important components which can improve sustainable development in the agricultural sector [1]. Further development of methods and techniques in the field of experimental practice can positively affect the process of achieving these requirements.

The sowing equipment used in experimental practice require high degree of modularity to use it for as many crop species as possible and to be able to assess as many issues as possible within the experimental research with respect to the optimisation of the seeding process. A prerequisite is an even distribution of the defined amount of seeds over the predetermined length of a trial plot. Depending on the explicit research question, the distances between the rows, which number can vary, need to be exactly retained. Above all an accurate and steady sowing depth, an important parameter for the optimum germination $[2,3]$, needs to be maintained for each row on the entire plot length and ideally should be carried out automatically. The mechanisation within the experimental praxis started in second half of the twentieth century and the backgrounds and requirements for sowing equipment have been listed and evaluated by Kemp [4], Cameron et al. [5] and Hergert [6]. In that time, considerable savings in time and labour were anticipated from the newly developed machinery in regarding the manual sowing.

Medicinal and aromatic crops belong to high value specialty crops and are often established on fields by sowing. The species vary in grain size, thousand seed weight, required seed rates, shape and surface textures, strength of the bran and composition of the grain sizes. These parameters particularly affect the performance of the single-grain seed drills, as well as seeders equipped with a cone-belt distributor. Experiments with finegrained aromatic and medicinal plants in which conventional seeders were used provided inadequate results [3].

The most of the modern agricultural machines are based on the functional principles presented in the past. However, the implementation of a powerful and often inexpensive electronics offered an expeditious development of new concepts, improvement in quality and efficiency, as well as introduction of newer, smarter ways in operator-machine interaction.

Considering the facts above and state of the art in both, the sowing technology and mechatronics, as well as the practical experiences of the employees of the Bavarian state research center for agriculture (LfL) within the field of sowing of medicinal and aromatic plants, an electrically driven precision plot seed drill was designed and built within the scope of a research project [7]. During the development the even lateral and longitudinal distribution of seeds, including fine-grained seeds regardless of the soil type and terrain configuration were particularly emphasised. Furthermore, a modular design was striven for allowing quick adaptation for sowing in shallow cultivation or rather on high dams with variable adjustable number of rows and row spacings. Additional task was to carry out the development harmonized with the Machinery Directive 2006 /42 / EC which inter alia regulates the technical requirements for electronically controlled machines. According to this topic, programmable control systems are primarily addressed within the regulation, because security strategies may be needed for agricultural and forestry mobile machines as well [8]. 


\section{Material and methods}

In the first phase a digital prototype of a sowing aggregate has been created using Autodesk Product Design Suite. The digital prototype was prepared for examining the characteristics under different conditions. In order to evaluate the functionality and assure optimisation already in the early phase of the development, kinematic simulations of the important mechanisms and force analysis has partly been carried out (see Figure 1). In the following phase a single module with a modified cone belt distributor ( $\mathrm{Fa}$. Wintersteiger) was built and as a mechanically driven functional model tested under real conditions. The objective was to compare the effects of a new design with the conventional coulter system. In parallel, electric actuators, sensors and controller were selected and tested under laboratory conditions to prepare the module for electrification. The electrification itself was based on a softwareoriented and modular approach and the implementation of off-theshelf, reusable components, in order to increase the efficiency of the system design. One of the biggest challenges within the field of machine development is the adoption of embedded technologies. The modular approach helps on the one hand to develop reusable components, but on the other hand significantly affects the way of the design process. Inter alia the modularity of the mechanical system needs to be reflected within the controller architecture. Although the traditional programmable logic controllers (PLCs) still play an important role within implementation in machine control systems, a decision was made to implement an embedded controller. The aim was to improve the flexibility, to assure the advantage of condition monitoring, to provide possibilities for easier upgrading than on systems with fixed logic (adaptability in term of changing I/O requirements), as well as to use advanced control techniques based on machine vision, and motion control capabilities, if necessary. The developed embedded controller is based on single-board RIO (sbRIO) platform [9] and electronic expansion circuit board for signal conditioning developed at the institute. Speed and resource trade-offs are typical issues related to programmable gate Array (FPGA)-based implementations. Generally, FPGA takes the benefits of the high execution speeds on FPGA targets but the software design requires minimisation of the resource consumption. Even with trends like multi-core, a processor-based solution will never get the parallelism offered by FPGAs [10].
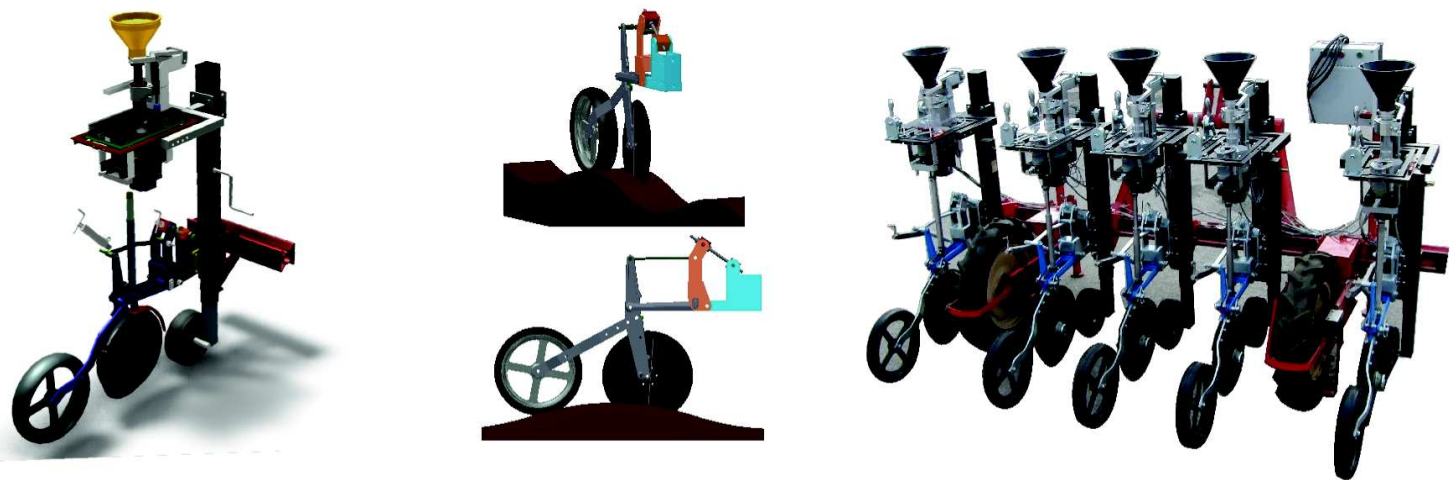

Figure 1. Digital prototype (left); example of a simulation with digital prototype (middle) and physical prototype of the precision plot seeder for medicinal and aromatic plants of the LfL with five electrically driven seeding

units and passive compensation of the slope inclination (right)

The complexity and the increasing number of questions and hypothesis within experimental praxis which are expected to be answered or tested require an ever increasing amount of field data. Inter alia data related to the presetting of the machine for each actual sowing scenario can be valuable, if possible shortcomings and impacts of those parameters on the expected sowing quality should be analysed. For example definition of the optimal parameters, setting and assessing the accuracy and efficiency with high temporal and longitudinal motion resolution can deliver completely new insights. Since development of a new seeder was aimed, the implementation of sbRIO offered the advantage of capturing and storing of all states, sensor and control signals with a correspondingly high sampling rate for later analysis, like in previous projects $[11,12]$. On such a way comprehensive documentation of the sowing process is secured. The control software was created using NI LabVIEW Developer Suite (Real-Time (RT) and FPGA modules).

The functionality of modern machines relies on an ever greater share of control software. Because of the increasing complexity the control software must be tested at an early stage of development to avoid risk of errors in the field. Conventional design methods in mechanical engineering provide only limited software testing opportunities before the physical machine is available. The fact, that a truly multidisciplinary approach to embedded system design requires not only simulation capability within domains of mechanical, control, electrical engineering and embedded computing but also synergistically combines these disciplines [13], is well known. As the cost of computing decrease and the amount of data increase, the likelihood that applications will fail due to numerical errors accumulate. Hence, a not fully deterministic testing software (model) running under Windows which emulates the physical system was created. The aim was to replicate the operating modes and use simulated signals in order to allow demonstration of the operating sequences to the user, test of the software prior to actual commissioning including intentionally implemented failures. In a further step a more rigorous full hardware-in-the-loop (HIL) testing was carried out whereas the sensors and actuators where implemented on a test bench, to test determinism and the dynamic performance of the prototype. Finally, test under real condition were carried out and documented.

\section{Results}

After development of a digital prototype and optimisation based and simulation five aggregates were built. The frame of an existing vegetable planter for small seeds (Gaspardo) was used and the newly developed aggregates adapted for individual and quick setup on it. The base frame of each aggregate and the carrier of the cone-belt distributor are designed to compensate the transversal and longitudinal slope based on a gyro mechanism. Additionally, a quick adaptation for sowing in the seedbed or on dams with different heights is provided. Special mechanism for automatic triggering of the lifting device, as well as at the 
individual and manual setting of the sowing depth and the coulter pressure for each unit are the most interesting feature of the novel seeder. The currently manual adjustment of the position of the gyro mechanism, of the sowing depth and of the coulter pressure can be automated. As furrow openers the practice-proven double disc coulters of the company Lemken were chosen (see Figure 1). The control electronics is housed in a robust enclosure, which at the same time has the functionality of the control panel and human-machine-interface (HMI). The control buttons and status indicators assure simple and clear structured control.

Each cone belt distributor is coupled to a stepper motor PD6N89 - Plug \& Drive (Nanotec). Stepper motors with drives are one of the most commonly used solutions for motion control. An accurate positioning without implementation of a feedback device such as encoder was possible. Depending on the used mode, up to 12800 steps per revolution can be configured. The main advantage is that a high torque can be reached already by relatively low rotational speeds. Additional advantage of the implemented motors is the method of its control. It can be carried out via network using an RS485 or CANopen protocol, or by providing the position, speed, or torque signals using separate digital inputs.

The electric drives, encoder MNI20 (Pepperl+Fuchs) and the controller were chosen and combined with the aim to provide high longitudinal sowing resolution. For the used vegetable planter which tires have a diameter of $680 \mathrm{~mm}$ one micro step of the drive corresponds to a motion of the seeder smaller than 1 $\mathrm{mm}$ in longitudinal direction. The main reason of choosing such a high accuracy is to minimise the losses of the expensive seed and to achieve higher accuracy in the longitudinal distribution. The speed of the stepper motor which drives the cone belt distribution is dynamically calculated based on the speed of the tractor and the length of the trial plot.

Typically, in case the software should intelligently respond to a stimulus such as sensor signals or HMI, using of state machines is the most powerful aspect in LabVIEW [14]. Since by using this design pattern the program execution is no longer linear, the part of the code which will be executed depends on the inputs from HMI or sensors (asynchronous discrete-time events), or the results of the current execution. Traditionally, FPGA design were limited to hardware designers, but using LabVIEW FPGA control systems designers are supported with enough "system-level" abstraction in order to deploy their designs to FPGAs [10]. Thus, a combination of an FPGA and RT software to control the sequences of the sowing cycle was developed in the form of a state machine, allowing to extend its functionality without restrictions. In the RT software sowing parameters (e.g. length of the trial plot, desired number of motor steps within one sowing cycle) can be read from the XML configuration file which is stored on a USB memory card. If required, all states of the digital inputs and outputs (I/O-s) and variables can be transmitted via FIFO from the FPGA software in RT software, to store them into a log file for further off-line analysis. One of the main drawbacks to use state machines is the difficulty to follow the program flow and because of this, debugging and tracing of errors can be in some cases tricky, particularly in time critical application where execution highlighting is not an option [14]. Thus, several models on a subsystem and system level were developed to carry out testing. The simulation models included the behaviour model, reproducing the logical behaviour of the machine and the kinematics model visualising the movement behaviour of the machine (see Figure 2).
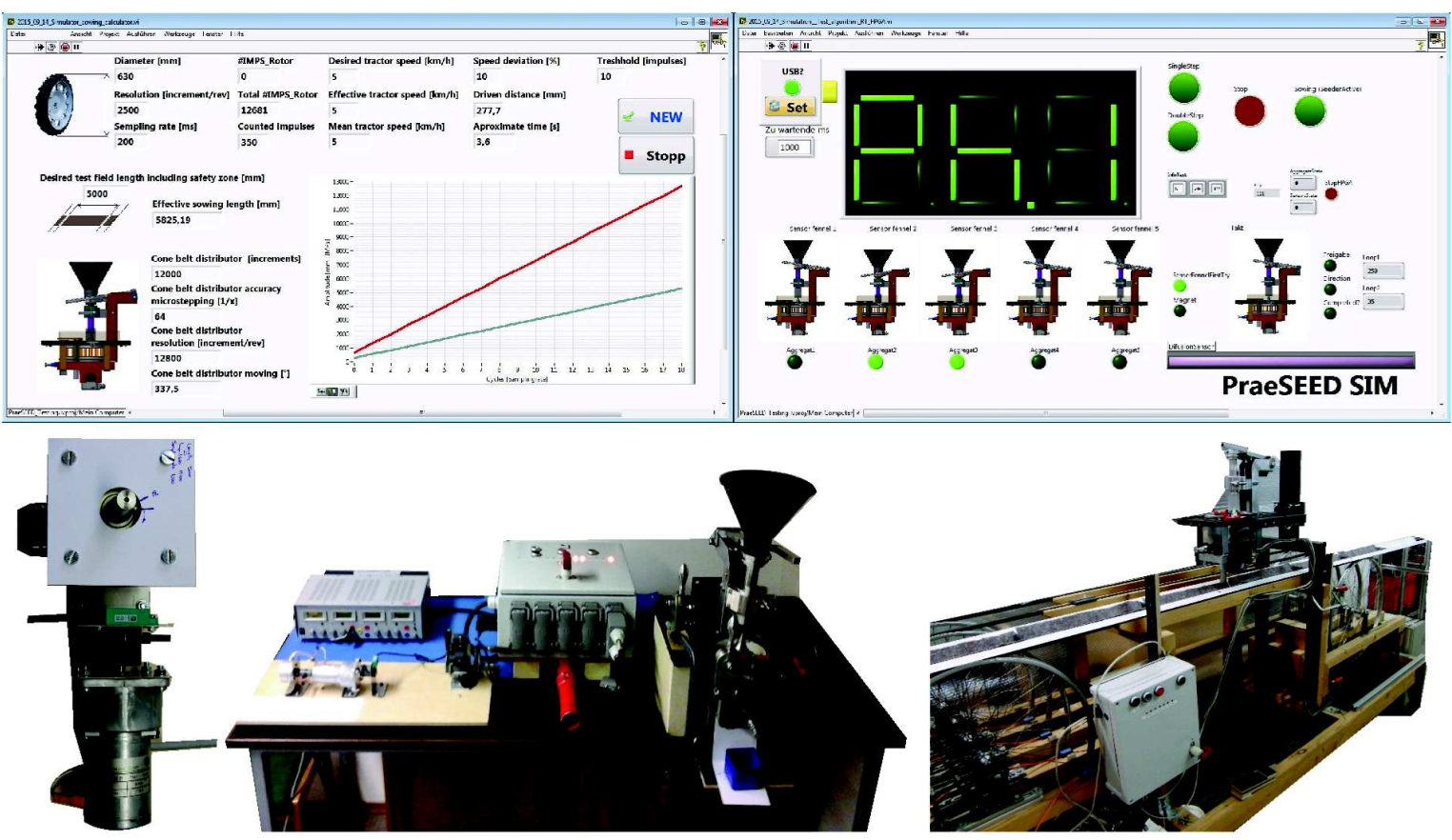

Figure 2. Front panel of the not fully deterministic testing software (simulation model) running under Windows which calculates the number steps (position) of the cone belt distributor depending on the simulated inputs (top left); front panel of the simulation model reproducing the logical behaviour of the seeder with visualisation of the movement behaviour (top right); sub-system level HIL test of the stepper motor (bottom left); HIL test of the system (bottom middle); testing of the longitudinal distribution on test bench (bottom right)

This method can be either used during development or for later revisions or bug fixes, just as well in cases when the access to the physical system or the real-time target is not possible nor desired. Applications written in G rely on more than just the compiled LabVIEW source code, meaning that it is impossible for each version of LabVIEW to produce the same numerical results as in a prior version $[15,16]$. Running the same application on different platforms, also if same version of LabVIEW is used, can generate irregularities in numerical results [14]. Hence it is necessary to carry out software testing before the commissioning. Considering 
the fact that the most system design errors are introduced in the original specification, but aren't found until the test phase, the early verification and validation techniques based on the models and virtual testing helped to detect the errors timely. Therefore, virtual commissioning of the control algorithm and FPGA and RT part of the software have been done by software in the loop (SIL) tests in virtual environment before uploading to the controller. (see Figure 2 top right). Further, the HIL simulation was carried out using a system-level model, which included the embedded system algorithm and the operating environment in form of a real-time simulator delivering inputs and receiving outputs from the embedded system as the real system would (see Figure 2 bottom middle). Using HIL simulation it was possible to test and verify the real-time performance of the embedded system. The main advantage of this method was a possibility to verify the logical behaviour in real time, to test operation and failure conditions that are difficult to physically replicate and to check and optimise the control software. For example the simulation showed that a manual or visual inspection may reveal the outage of one or more of the connected fennels in every sowing cycle, but this solution is suboptimal considering the number of rows and number of experiments which usually needs to be carried out in a short time consecutively. Thus, the controlling algorithm was designed to carry out self-diagnostic and if there is no outage indication, the control algorithm can move into the next stage without causing damage within the hardware or impeding the consistent transversal distribution.

Virtual commissioning allowed to test the performance under different scenarios and recognise the problems in the development stages which resulted in less debugging during the commissioning phase. In the post-processing phase of the virtual commissioning the testing results were analysed in order to implement changes and document the possible problems and differences in relation to the real installation on the prototype. Unfortunately, the experiences from practice showed that during the implementation phase additional errors and difficulties can occur which have not been detected within modelling and simulation phase because during the commissioning of a real installation mostly different topics merge. Particularly, new concerns can be induced: trough installations requiring clarification of the components functionality; since the fact that software is always the last thing installed and its development during the commissioning is common; trough parameterisation and optimisation of the software during this phase; through errors caused by unintended network communication; as well as the acceptance test and the introduction of the novel product to the operators and the service personnel.

\section{Conclusion}

Historically, engineers of different disciplines worked in their departments on specific task, carrying out sequential development and making design decisions independently, resulting in longer development cycles. Today, considering the mechatronic approach the streamline is parallel development and collaboration of several teams on design, prototyping, and deployment. The fundamental aspect is to create virtual prototypes in order to explore the machines before they are built. In conventional manufacturing the commissioning is usually one of the last steps before the machine is delivered to the customer. In case, shortcomings are first realised in this phase of the project, design correction are required, causing sometimes exponential increase of the costs and undesirable changes in the time schedules.

The digital prototype enabled both the functional test of the newly developed mechanisms and assemblies, as well as analyses based on simulation. Possible design deficits were minimised through optimisation based on analysis of gained results and significantly reduced the time for the development and assembling the prototype. The tests showed a high stability and robustness of the mechanics and provided basis for the further development including electrification of the prototype (Figure 3.). The electrification was carried out based on a software-oriented and modular approach, using off-the-shelf reusable components. Thus, the efficiency of the system design was enhanced.

Using simulations with created system models, which included the FPGA and RT software models, additional insight were gained about the system behaviour, the human machine interface (HMI) and the physical and environmental aspects of the sowing process. System models allowed defining different simulation scenarios and documenting of the behaviour of the control algorithm and its systematic analyses. The analysis can reflect the completeness and integrity of the software regarding of requirements and also can be used for further design refinement. During the development design of reusable components including mechanical parts and software was aimed, in order to create and test mechatronic components in terms of modules or parts of a test bench which can be used in further projects. Both, the SIL and the HIL test allowed optimisation of the HMI based on the operators' feedback, as well as the possibility to learn the operations and how fault conditions are indicated and handled within a safe simulation environment.

The experiments under controlled conditions and virtual commissioning, only partially led to successful commissioning within the implementation phase, because only observations in a finite number of predefined and generally simplified scenarios can be carried out under such conditions. Hence, enough data needs to be collected during field tests under uncontrolled conditions to possibly identify the problematic scenarios which may not have been considered during the machine development or rather the design of simulations. By this means events and effects which couldn't be anticipated or observed previously could be explained.
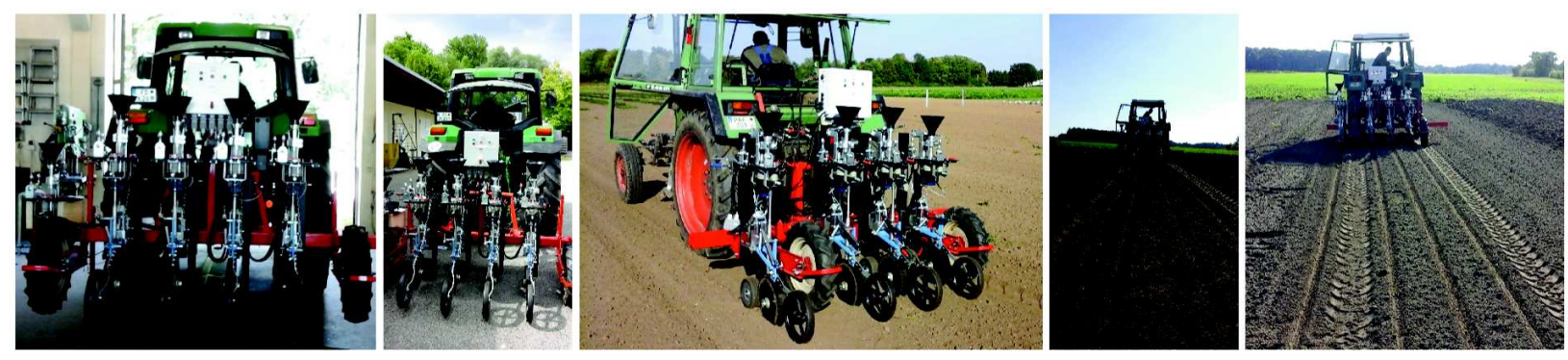

Figure 3. Testing of the precision plot seeder for medicinal and aromatic plants of the LfL with electrically driven seeding units and passive compensation of the slope inclination 


\section{Acknowledgements}

The authors thank to all involved employees of the Institute for Agricultural Engineering and Animal Husbandry, the Institute for Crop Science and Plant Breeding and experimental stations of the Bavarian State Research Center for Agriculture for the excellent cooperation.

\section{References}

[1.] OECD/FAO 2012. OECD-FAO Agricultural Outlook 20122021, OECD Publishing and FAO. http://dx.doi.org/ 10.1787/19991142

[2.] Ziegler K., Göbel E., Schmittmann 0., Schulze Lammers P.: 2012. Precision seed drill tests in Germany 2009-2011. International sugar journal 114/1367, 795-803

[3.] Meinhold T., Blum H., Budde M., Damerow L., Schulze Lammers P.: 2013. Partial Process-Analysis of the Sowing Process for Fine Seeds. Conference proceedings Land. TechnikAgEng 2013, 8.9. Nov. 2013 Hannover, 189-195. [4.] Kemp, D. C.: 1966. A brassica plot seeder. Journal of Agricultural Engineering Research, 11 (2). pp. 135147. http://dx.doi.org/10.1016/S0021-8634(66)80051-3

[5.] Cameron D., Milner J. B., Carruthers J.: 1967. An automatic self-propelled seed drill for cereal plots. Journal of Agricultural Engineering Research, 12 (2), 142-146. http://dx.doi.org/10.1016/S0021-8634(67)80008-8

[6.] Hergert G. B.: 1969. A spaced-plant seeder for cereal plots. Canadian Journal of Plant Science, 49, 642-644.

[7.] Gobor Z., Heuberger H., Rinder R.:

2014. Entwicklung und Optimierung einer

Präzisionssämaschine für Heil- und Gewürzpflanzen

(Feinsämereien). Mitteilung der Gesellschaft für

Pflanzenbauwissenschaften Band 26 ISSN:0934-5116 pp.323-

324
[8.] Zeltwanger, H.: 2013. Safety and security requirements in mobile machines, Proceedings, 1st international Mobile Machine Control (MMC) conference, Nuremberg, Germany, 12-13. Jun 2013, CAN in Automation GmbH, pp. 01-7 - 01-12

[9.] National Instruments 2014. OEM OPERATING INSTRUCTIONS AND SPECIFICATIONS NI sbRIO9605/9606 and NI sbRIO-9623/9626/9633/9636

[10.] Kassas Z. M.: 2011. Methodologies for Implementing FPGA-Based Control Systems. Proceedings of the 18th IFAC World Congress; Milano, Italy. 28 August-2 September 2011

[11.] Gobor Z.: 2013. Data acquisition and automated data analysis in prototype development - Testing of the prototype for automated attachment of the supporting wires in hop gardens. 1st international Mobile Machine

[12.] Gobor Z.: 2015. Development of a diagnostic tool for performance analysis during the testing of agricultural implements. SCILABTEC 2015 7th International scilab user conference. Paris 21-22.05.2015

[13.] MacCleery B., Kassas Z. M.: 2008. New mechatronics development techniques for FPGA-based control and simulation of electromechanical systems. Proceedings of the 17th IFAC World Congress; Seoul, Korea. 6-11 July 2008

[14.] Bitter R., Mohiuddin T., Nawrocki M.: 2007. LabVIEW: Advanced Programming Techniques, Second Edition, CRC Press, Boca Raton London New York

[15.] Farkas I., Rendik Z.: 1997. Intermittent thin layer corn drying, Drying Technology, Vol. 15, pp. 1951-1963. http://dx.doi.org/10.1080/07373939708917340

[16.] Schmidt D.: 2010. An Introduction to Floating-Point Behavior in LabVIEW. National Instruments Corporation. 